\title{
Analysis of fractional partial differential equations by Taylor series expansion
}

\author{
Ali Demir ${ }^{{ }^{*}}$, Sertaç Erman ${ }^{1}$ Berrak Özgür ${ }^{1}$ and Esra Korkmaz²
}

\author{
"Correspondence: \\ ademir@kocaeli.edu.tr \\ ${ }^{1}$ Department of Mathematics, \\ Kocaeli University, Umuttepe, Izmit, \\ Kocaeli 41380, Turkey \\ Full list of author information is \\ available at the end of the article
}

\begin{abstract}
We develop a formulation for the analytic or approximate solution of fractional differential equations (FDEs) by using respectively the analytic or approximate solution of the differential equation, obtained by making fractional order of the original problem integer order. It is shown that this method works for FDEs very well. The results reveal that it is very effective and simple in determination of solutions of FDEs.
\end{abstract}

\section{Introduction}

Fractional differential equations (FDEs) are obtained by generalizing differential equations to an arbitrary order. Since fractional differential equations are used to model complex phenomena, they play a crucial role in engineering, physics and applied mathematics. Therefore they have been generating increasing interest from engineers and scientist in recent years. Since FDEs have memory, nonlocal relations in space and time, complex phenomena can be modeled by using these equations. Due to this fact, materials with memory and hereditary effects, fluid flow, rheology, diffusive transport, electrical networks, electromagnetic theory and probability, signal processing, and many other physical processes are diverse applications of FDEs [1-7].

In [8], the solutions for some nonlinear fractional differential equations are constructed by using symmetry analysis. But, in general, FDEs do not have exact analytic solutions, hence the approximate and numerical solutions of these equations are studied. Analytical approximations of linear and nonlinear FDEs are obtained by the variational iteration method, Adomian's decomposition method, the homotopy perturbation method and the Lagrange multiplier method [9-24].

In the present paper, we use the Taylor series of an analytical solution for the differential equations which is obtained from FDEs by making the fractional order of the derivative integer, to obtain the analytical or approximate solution of FDEs. We can obtain the exact or approximate solution of FDEs by changing the terms of Taylor series expansion for a solution of a differential equation in such a way that the relationship among the terms of Taylor series expansion in the sense of derivative and fractional derivative remains the same. Applications of this method show that it is easy and effective when applied to any FDEs as long as the differential equation obtained from FDEs has an analytical or approximate solution. We take the fractional derivative in the Caputo sense.

The structure of this article is as follows. In Section 2, we give the construction of analytical or approximate solutions for FDEs including fractional derivative with respect to 
time. In the same manner, we obtain the analytical or approximate solution of FDEs with fractional derivative with respect to space variable in Section 3. In Section 4, we take the combination of previous two sections, and we get the analytical or approximate solution of FDEs with fractional derivative with respect to time and space variable. Finally, we give some illustrative examples of this method for all cases in Section 5.

\section{Solution of FDEs including fractional derivative with respect to time}

Let us consider the following FDE:

$$
D_{t}^{\alpha} u(x, t)=F\left(u, \frac{\partial u}{\partial x} \cdots \frac{\partial^{n} u}{\partial x^{n}}, x, t\right), \quad m-1<\alpha \leq m, t>0 .
$$

In order to determine the solution of this equation, we first need to determine the solution of the following differential equation:

$$
D_{t}^{m} u(x, t)=F\left(u, \frac{\partial u}{\partial x} \cdots \frac{\partial^{n} u}{\partial x^{n}}, x, t\right), \quad t>0,
$$

which is obtained by taking $\alpha=m$. After finding an analytic or approximate solution of equation (2), we can obtain the exact or approximate solution of equation (1) by changing the terms of Taylor series expansion for the solution of differential equation (2) in such a way that the relationship among the terms of Taylor series expansion in the sense of derivative and fractional derivative with respect to time remains the same. In other words, we expand the exact or approximate solution into its Taylor series with respect to $t$. Then we replace the derivatives with respect to $t$ by fractional derivatives with respect to $t$ in such a way that the relation among the terms of Taylor series is preserved. Moreover, we leave the first $m$ terms of Taylor series fixed since $\alpha=m$. This also allows the solution of the fractional differential equation to satisfy the initial conditions of the problem. Let us assume that the solution of equation (2) is expanded into its Taylor series with respect to $t$ as follows:

$$
u(x, t)=\sum_{n=0}^{\infty} \frac{\partial^{n} u(x, 0)}{\partial t^{n}} \frac{t^{n}}{n !} .
$$

Then the solution of equation (1) becomes

$$
\begin{aligned}
u(x, t)= & \sum_{n=0}^{m-1} \frac{\partial^{n} u(x, 0)}{\partial t^{n}} \frac{t^{n}}{n !} \\
& +\sum_{n=1}^{\infty} \sum_{i=0}^{m-1} \frac{\partial^{m n+i} u(x, 0)}{\partial t^{m n+i}} \frac{t^{n \alpha+i}}{\Gamma(n \alpha+i+1)} .
\end{aligned}
$$

For instance, let us assume that $0<\alpha \leq 1$ and the solution of equation (2) is $u(x, t)=e^{x} e^{t}$. In order to find the solution of equation (1), we expand this solution into Taylor series with respect to $t$ as follows:

$$
u(x, t)=\sum_{n=0}^{\infty} \frac{e^{x} t^{n}}{n !} .
$$



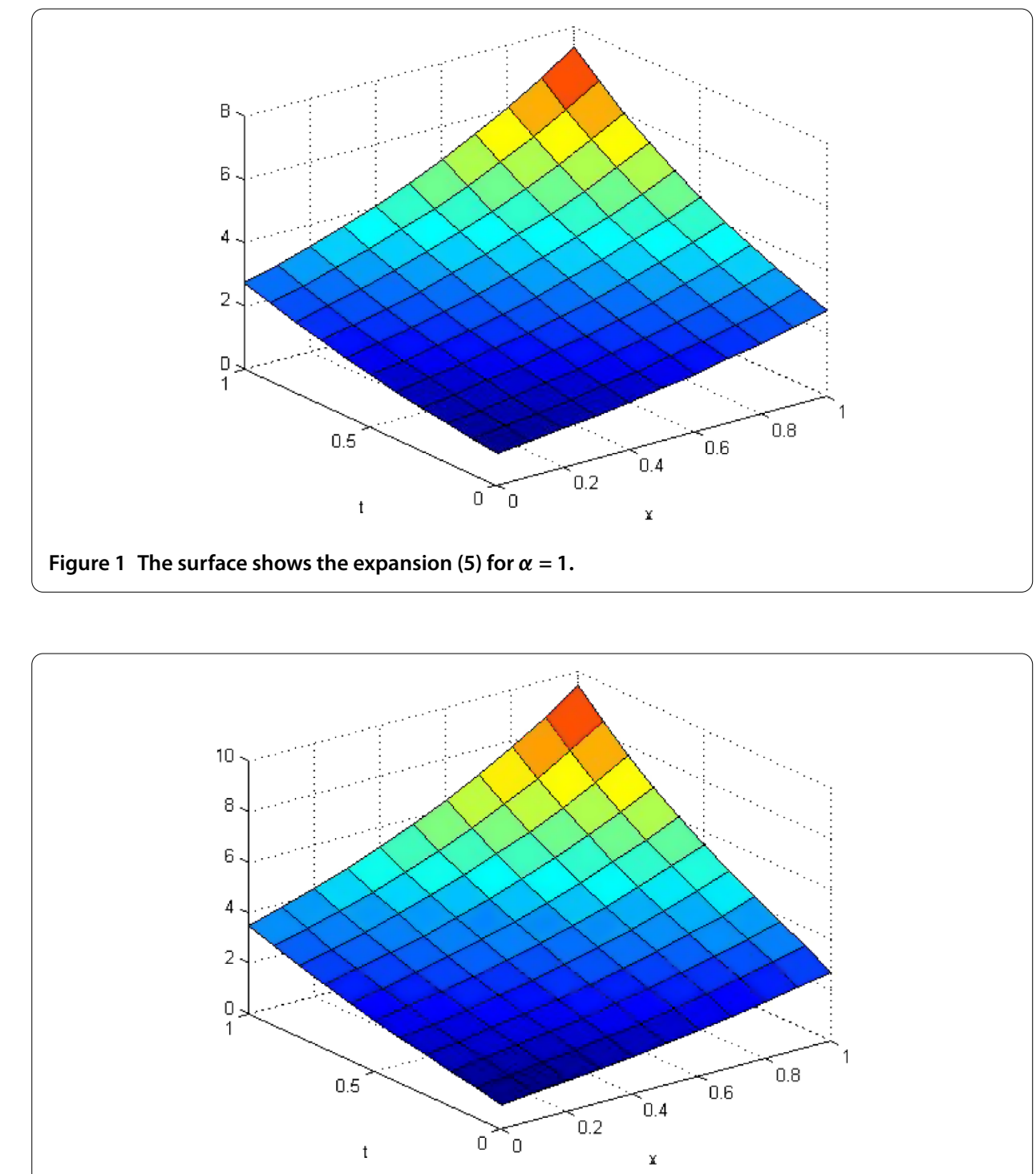

Figure 2 The surface shows the expansion (5) for $\alpha=0.75$.

Based on (4), the solution of equation (1) can be written in the following form:

$$
u(x, t)=e^{x}+\frac{e^{x} t^{\alpha}}{\Gamma(\alpha+1)}+\frac{e^{x} t^{2 \alpha}}{\Gamma(2 \alpha+1)}+\frac{e^{x} t^{3 \alpha}}{\Gamma(3 \alpha+1)}+\cdots
$$

Figures 1-3 show the evolution results for the expansion (5) obtained for different values of $\alpha$.

\section{Solution of FDEs including fractional derivative with respect to space variable}

Let us consider the following FDE:

$$
D_{x}^{\alpha} u(x, t)=F\left(u, \frac{\partial u}{\partial t} \cdots \frac{\partial^{n} u}{\partial t^{n}}, x, t\right), \quad m-1<\alpha \leq m, t>0 .
$$




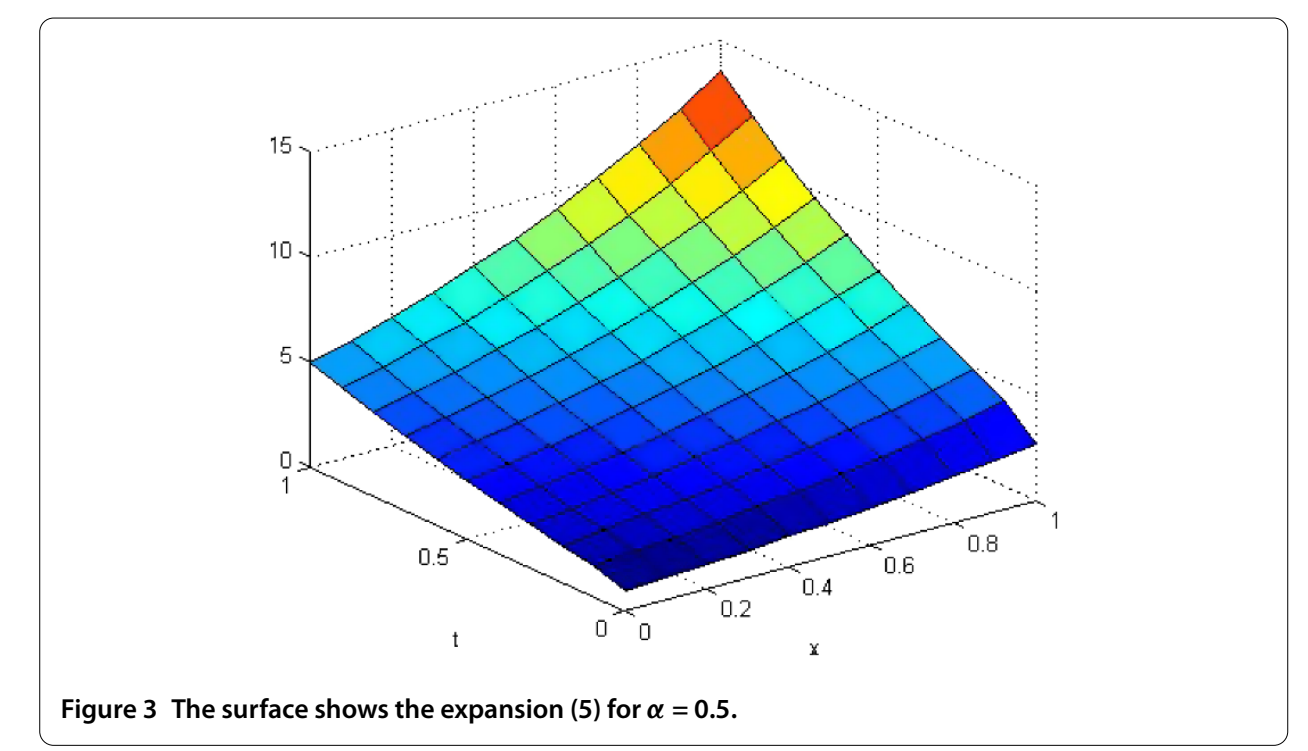

In order to determine the solution of this equation, we first need to determine the solution of the following differential equation:

$$
D_{x}^{m} u(x, t)=F\left(u, \frac{\partial u}{\partial t} \cdots \frac{\partial^{n} u}{\partial t^{n}}, x, t\right), \quad t>0,
$$

which is obtained by taking $\alpha=m$.

After finding the analytic or approximate solution of equation (7), we can obtain the exact or approximate solution of equation (6) by changing the terms of Taylor series expansion for the solution of differential equation (7) in such a way that the relationship among the terms of Taylor series expansion in the sense of derivative and fractional derivative with respect to space remains the same. In other words, we expand the exact or approximate solution into its Taylor series with respect to $x$. Then we replace the derivatives with respect to $x$ by fractional derivatives with respect to $x$ in such a way that the relation among the terms of Taylor series is preserved. Moreover, we leave the first $m$ terms of Taylor series fixed since $\alpha=m$. This also allows the solution of the fractional differential equation to satisfy the boundary conditions of the problem.

Let us assume that the solution of equation (7) is expanded into its Taylor series with respect to $x$ as follows:

$$
u(x, t)=\sum_{n=0}^{\infty} \frac{\partial^{n} u(0, t)}{\partial x^{n}} \frac{x^{n}}{n !}
$$

Then the solution of equation (6) becomes

$$
u(x, t)=\sum_{n=0}^{m-1} \frac{\partial^{n} u(0, t)}{\partial x^{n}} \frac{x^{n}}{n !}+\sum_{n=1}^{\infty} \sum_{i=0}^{m-1} \frac{\partial^{m n+i} u(0, t)}{\partial x^{m n+i}} \frac{x^{n \alpha+i}}{\Gamma(n \alpha+i+1)} .
$$

For instance, let us assume that $0<\alpha \leq 1$ and the solution of equation (7) is $u(x, t)=e^{t} e^{x}$. In order to find the solution of equation (6), we expand this solution into Taylor series with 
respect to $x$ as follows:

$$
u(x, t)=\sum_{n=0}^{\infty} \frac{e^{t} x^{n}}{n !}
$$

Based on (9), the solution of equation (6) can be written in the following form:

$$
u(x, t)=e^{t}+\frac{e^{t} x^{\alpha}}{\Gamma(\alpha+1)}+\frac{e^{t} x^{2 \alpha}}{\Gamma(2 \alpha+1)}+\frac{e^{t} x^{3 \alpha}}{\Gamma(3 \alpha+1)}+\cdots
$$

Figures 4-6 show the evolution results for the expansion (10) obtained for different values of $\alpha$.

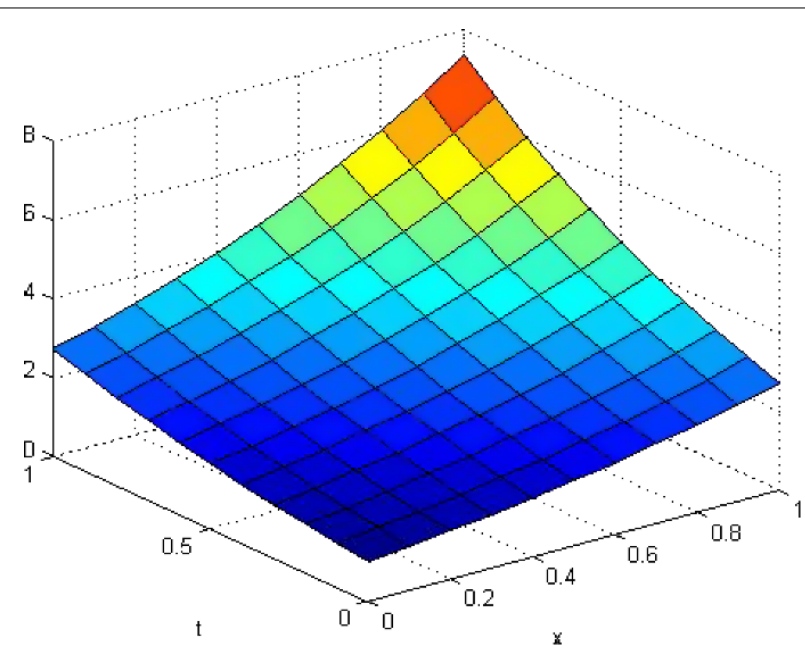

Figure 4 The surface shows the expansion (10) for $\alpha=1$.

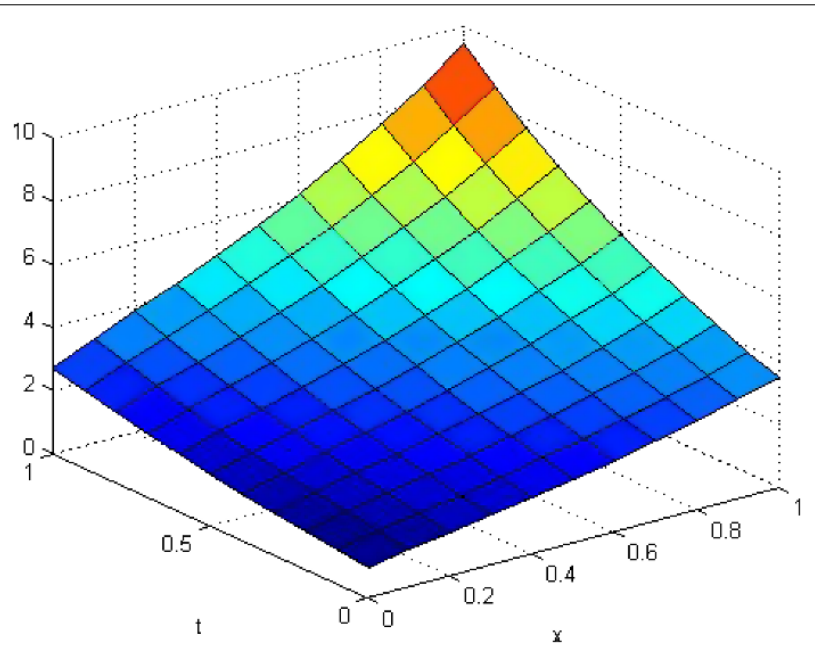

Figure 5 The surface shows the expansion (10) for $\alpha=0.75$. 


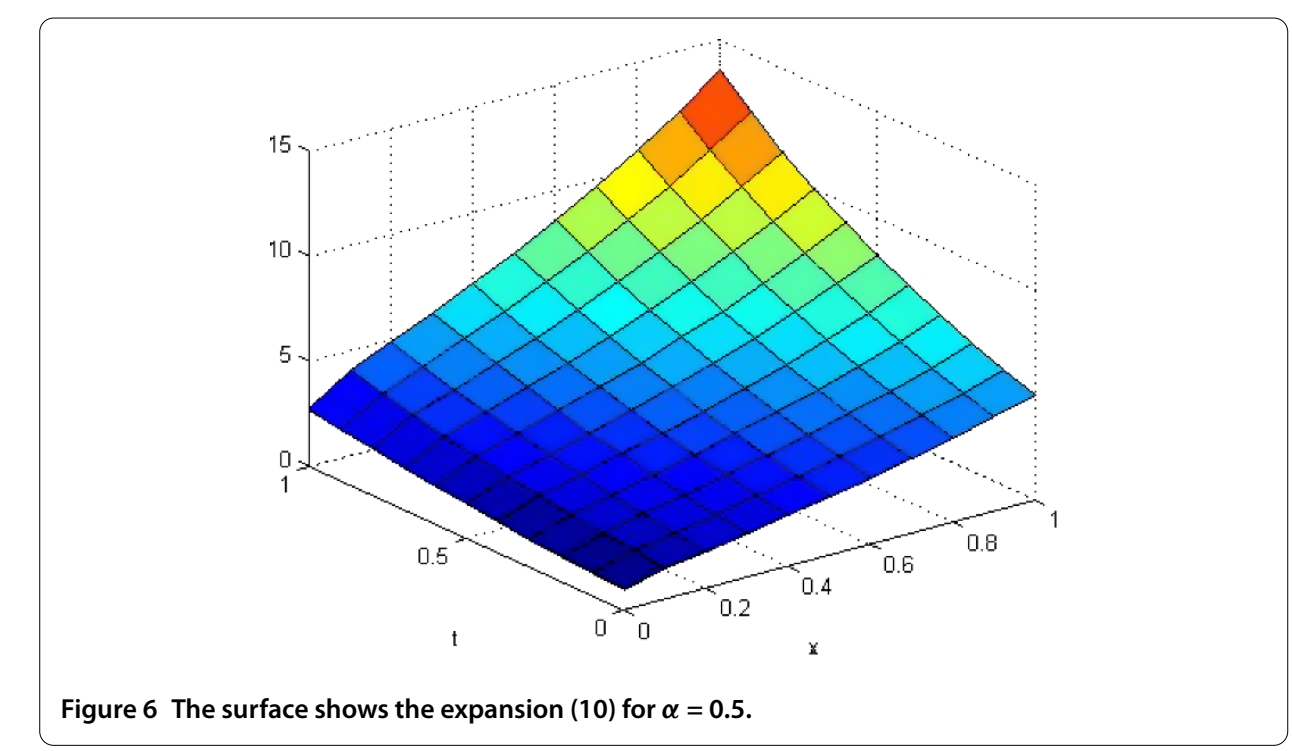

\section{Solution of FDEs including fractional derivative with respect to space variable and time}

Let us consider the following linear FDE with constant coefficients:

$$
F\left(u, D_{t}^{\beta} u, D_{x}^{\alpha} u, x, t\right)=0, \quad k-1<\beta \leq k, m-1<\alpha \leq m, t>0 .
$$

In order to determine the solution of this equation, we first need to determine the solution of the following differential equation:

$$
F\left(u, D_{t}^{k} u, D_{x}^{m} u, x, t\right)=0, \quad t>0,
$$

which is obtained by taking $\beta=k, \alpha=m$. Since it is a linear partial differential equation, its solution can be written as

$$
u(x, t)=u_{0}(x) u_{1}(t)
$$

After finding the analytic or approximate solution of equation (12), we expand $u_{0}(x)$ into its Taylor series with respect to $x$ and $u_{1}(t)$ into its Taylor series with respect to $t$. Then we replace the derivatives with respect to $x$ by fractional derivatives with respect to $x$ in $u_{0}(x)$ in such a way that the relation among the terms of Taylor series is preserved. Moreover, we leave the first $m$ terms of Taylor series fixed since $\alpha=m$. Similarly, we do the same thing for $u_{1}(t)$. This also allows the solution of the fractional differential equation to satisfy the initial and boundary conditions of the problem. Let us assume that $u_{0}(x)$ of equation (12) is expanded into its Taylor series with respect to $x$ as follows:

$$
u_{0}(x)=\sum_{n=0}^{\infty} \frac{\partial^{n} u_{0}(0)}{\partial x^{n}} \frac{x^{n}}{n !}
$$


Then $u_{0}(x)$ of solution (13) becomes

$$
\begin{aligned}
u_{0}(x)= & \sum_{n=0}^{m-1} \frac{\partial^{n} u_{0}(0)}{\partial x^{n}} \frac{x^{n}}{n !} \\
& +\sum_{n=1}^{\infty} \sum_{i=0}^{m-1} \frac{\partial^{m n+i} u_{0}(0)}{\partial x^{m n+i}} \frac{x^{n \alpha+i}}{\Gamma(n \alpha+i+1)} .
\end{aligned}
$$

Let us assume that $u_{1}(t)$ of equation (12) is expanded into its Taylor series with respect to $t$ as follows:

$$
u_{1}(t)=\sum_{n=0}^{\infty} \frac{\partial^{n} u_{1}(0)}{\partial t^{n}} \frac{t^{n}}{n !}
$$

Then $u_{1}(t)$ of solution (13) becomes

$$
\begin{aligned}
u_{1}(t)= & \sum_{n=0}^{m-1} \frac{\partial^{n} u_{1}(0)}{\partial t^{n}} \frac{t^{n}}{n !} \\
& +\sum_{n=1}^{\infty} \sum_{i=0}^{m-1} \frac{\partial^{m n+i} u_{1}(0)}{\partial t^{m n+i}} \frac{t^{n \alpha+i}}{\Gamma(n \alpha+i+1)}
\end{aligned}
$$

For instance, let us assume that $0<\beta \leq 1,0<\alpha \leq 1$, and that the solution of equation (12) is $u(x, t)=e^{x} e^{t}$. In order to find the solution of equation (11), we first expand $u_{0}(x)=e^{x}$ into Taylor series with respect to $x$ as follows:

$$
u_{0}(x)=\sum_{n=0}^{\infty} \frac{x^{n}}{n !}
$$

Similarly,

$$
u_{1}(t)=\sum_{n=0}^{\infty} \frac{t^{n}}{n !} .
$$

Based on (4) and (9), the expansions of $u_{0}(x)$ and $u_{1}(t)$ can be written in the following form:

$$
\begin{aligned}
& u_{0}(x)=1+\frac{x^{\alpha}}{\Gamma(\alpha+1)}+\frac{x^{2 \alpha}}{\Gamma(2 \alpha+1)}+\frac{x^{3 \alpha}}{\Gamma(3 \alpha+1)}+\cdots \\
& u_{1}(t)=1+\frac{t^{\beta}}{\Gamma(\beta+1)}+\frac{t^{2 \beta}}{\Gamma(2 \beta+1)}+\frac{t^{3 \beta}}{\Gamma(3 \beta+1)}+\cdots
\end{aligned}
$$

and then the solution of equation (11) can be written in the following form:

$$
\begin{aligned}
u(x, t)= & \left(1+\frac{x^{\alpha}}{\Gamma(\alpha+1)}+\frac{x^{2 \alpha}}{\Gamma(2 \alpha+1)}+\frac{x^{3 \alpha}}{\Gamma(3 \alpha+1)}+\cdots\right) \\
& \times\left(1+\frac{t^{\beta}}{\Gamma(\beta+1)}+\frac{t^{2 \beta}}{\Gamma(2 \beta+1)}+\cdots\right) .
\end{aligned}
$$




\section{Illustrative applications}

Example 1 Let us consider the following time-fractional initial boundary value problem:

$$
\begin{aligned}
& D_{t}^{\alpha} u(x, t)=\frac{1}{2} x^{2} u_{x x}(x, t), \quad 0<\alpha \leq 1, t>0, \\
& u(x, 0)=x^{2}, \quad u(0, t)=0, \quad u(1, t)=e^{t} .
\end{aligned}
$$

The exact solution, for the special case $\alpha=1$, is given by

$$
u(x, t)=x^{2} e^{t}
$$

Now we can apply series (4) to construct the solution $u(x, t)$ for $0<\alpha \leq 1$, and we have

$$
u(x, t)=x^{2}\left[1+\frac{t^{\alpha}}{\Gamma(\alpha+1)}+\frac{t^{2 \alpha}}{\Gamma(2 \alpha+1)}+\frac{t^{3 \alpha}}{\Gamma(3 \alpha+1)}+\cdots\right]
$$

which is exactly the same solution as in [18].

Example 2 Let us consider the following time-fractional initial boundary value problem:

$$
\begin{aligned}
& D_{t}^{\alpha} u=u_{x x}(x, t)+x u_{x}(x, t)+u(x, t), \quad 0<\alpha \leq 1, t>0, \\
& u(x, 0)=x, \quad u_{x}(x, 0)=1, \quad u(0, t)=0 .
\end{aligned}
$$

The exact solution, for the special case $\alpha=1$, is given by

$$
u(x, t)=x e^{2 t} .
$$

Now we can apply series (4) to $u(x, t)$ for $0<\alpha \leq 1$, and we have

$$
u(x, t)=x^{2}\left[1+\frac{2 t^{\alpha}}{\Gamma(\alpha+1)}+\frac{4 t^{2 \alpha}}{\Gamma(2 \alpha+1)}+\frac{8 t^{3 \alpha}}{\Gamma(3 \alpha+1)}+\cdots\right]
$$

which is exactly the same solution as in [19].

Example 3 Let us consider the nonlinear time-fractional Fisher's equation

$$
\begin{aligned}
& D_{t}^{\alpha} u=u_{x x}(x, t)+6 u(x, t)(1-u(x, t)), \quad 0<\alpha \leq 1, t>0, \\
& u(x, 0)=\frac{1}{\left(1+e^{x}\right)^{2}} .
\end{aligned}
$$

The exact solution, for the special case $\alpha=1$, is given by

$$
u(x, t)=\frac{1}{\left(1+e^{x-5 t}\right)^{2}} .
$$

As in the previous examples, we can apply series (4) to obtain the solution $u(x, t)$ for $0<\alpha \leq 1$, and we get

$$
u(x, t)=\frac{1}{\left(1+e^{x}\right)^{2}}+\frac{10 e^{x}}{\left(1+e^{x}\right)^{3}} \frac{t^{\alpha}}{\Gamma(\alpha+1)}+\frac{50 e^{x}\left(-1+2 e^{x}\right)}{\left(1+e^{x}\right)^{4}} \frac{t^{2 \alpha}}{\Gamma(2 \alpha+1)}+\cdots,
$$

which is totaly the same solution as in [20]. 
Example 4 Let us consider the following time-fractional initial boundary value problem:

$$
\begin{aligned}
& D_{t}^{\alpha} u= \pm u_{x x}(x, t), \quad 0<\alpha \leq 1, t>0, \\
& u(x, 0)=x^{2}, \quad u(0, t)=\frac{2 k t^{\alpha}}{\Gamma(\alpha+1)}, \quad u(\ell, t)=\ell^{2}+\frac{2 k t^{\alpha}}{\Gamma(\alpha+1)},
\end{aligned}
$$

where the boundary conditions are given in fractional terms.

Boundary value problem (17)-(18), for the special case $\alpha=1$, becomes as follows:

$$
\begin{aligned}
& u_{t}= \pm u_{x x}(x, t), \quad 0<\alpha \leq 1, t>0, \\
& u(x, 0)=x^{2}, \quad u(0, t)=2 k t, \quad u(\ell, t)=\ell^{2}+2 k t,
\end{aligned}
$$

and its analytic solution is obtained as follows:

$$
u(x, t)=x^{2}+2 k t .
$$

Now we can apply series (4) to $u(x, t)$ for $0<\alpha \leq 1$, and we have

$$
u(x, t)=x^{2}+2 k \frac{t^{\alpha}}{\Gamma(\alpha+1)},
$$

which is exactly the same solution as in [21].

Example 5 Let us consider the following space-fractional initial boundary value problem:

$$
\begin{aligned}
& u_{t}(x, t)=D_{x}^{\beta} u(x, t)-(1+\tan t) u(x, t), \quad 1<\beta \leq 2, \\
& u(x, 0)=e^{x}, \quad u(0, t)=\cos t, \quad u_{x}(0, t)=\cos t .
\end{aligned}
$$

The exact solution, for the special case $\beta=2$, is given by

$$
u(x, t)=e^{x} \cos t .
$$

Now we can apply series (9) to $u(x, t)$ for $1<\beta \leq 2$, then we have

$$
u(x, t)=\cos t\left(1+x+\frac{x^{\beta}}{\Gamma(\beta+1)}+\frac{x^{\beta+1}}{\Gamma(\beta+2)}+\frac{x^{2 \beta}}{\Gamma(2 \beta+1)}+\frac{x^{2 \beta+1}}{\Gamma(2 \beta+2)}+\cdots\right) .
$$

Example 6 Let us consider the following space and time-fractional initial boundary value problem:

$$
\begin{aligned}
& D_{t}^{\alpha} u(x, t)=2 D_{x}^{\beta} u(x, t)+\cos t, \quad 0<\alpha \leq 1,1<\beta \leq 2, \\
& u(x, 0)=e^{x}, \quad u(0, t)=e^{2 t}+\sin t, \quad u_{x}(0, t)=e^{2 t}+\sin t .
\end{aligned}
$$

The exact solution, for the special case $\alpha=1$ and $\beta=2$, is given by

$$
u(x, t)=e^{2 t} e^{x}+\sin t
$$


Now we can apply the series (4)-(9) to $u(x, t)$ for $0<\alpha \leq 1$ and $1<\beta \leq 2$, then we have

$$
\begin{aligned}
u(x, t)= & \left(1+\frac{2 t^{\alpha}}{\Gamma(\alpha+1)}+\frac{4 t^{2 \alpha}}{\Gamma(2 \alpha+1)}+\cdots\right)\left(1+x+\frac{x^{\beta}}{\Gamma(\beta+1)}+\frac{x^{\beta+1}}{\Gamma(\beta+2)}+\cdots\right) \\
& +\left(\frac{t^{\alpha}}{\Gamma(\alpha+1)}-\frac{t^{3 \alpha}}{\Gamma(3 \alpha+1)}+\frac{t^{5 \alpha}}{\Gamma(5 \alpha+1)}-\cdots\right) .
\end{aligned}
$$

Example 7 Let us consider the following space and time-fractional initial boundary value problem:

$$
\begin{aligned}
& D_{t}^{\alpha} u(x, t)=2 D_{x}^{\beta} u(x, t)+2 \sin x, \quad 0<\alpha \leq 1,1<\beta \leq 2, \\
& u(x, 0)=e^{x}+\sin x, \quad u(0, t)=e^{2 t}, \quad u_{x}(0, t)=e^{2 t}+1 .
\end{aligned}
$$

The exact solution, for the special case $\alpha=1$ and $\beta=2$, is given by

$$
u(x, t)=e^{2 t} e^{x}+\sin x
$$

Now we can apply the series (4)-(9) to $u(x, t)$ for $0<\alpha \leq 1$ and $1<\beta \leq 2$, then we have

$$
\begin{aligned}
u(x, t)= & \left(1+\frac{2 t^{\alpha}}{\Gamma(\alpha+1)}+\frac{4 t^{2 \alpha}}{\Gamma(2 \alpha+1)}+\cdots\right)\left(1+x+\frac{x^{\beta}}{\Gamma(\beta+1)}+\frac{x^{\beta+1}}{\Gamma(\beta+2)}+\cdots\right) \\
& +\left(x-\frac{x^{\beta+1}}{\Gamma(\beta+2)}+\frac{x^{2 \beta+1}}{\Gamma(2 \beta+2)}-\frac{x^{3 \beta+1}}{\Gamma(3 \beta+2)}-\cdots\right) .
\end{aligned}
$$

Figures 7-9 show the evolution results for the approximate solutions of problem (23)-(24) obtained for different values of $\alpha$ and $\beta$.

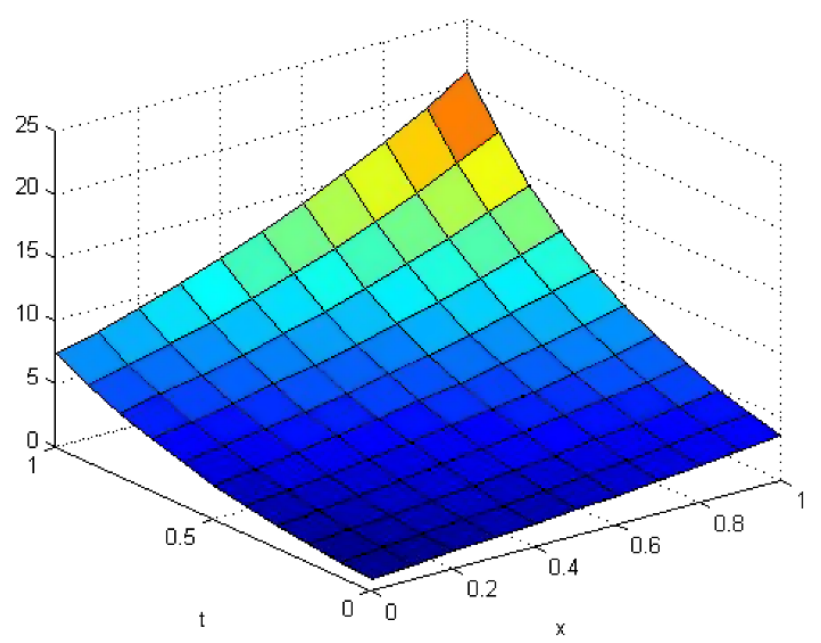

Figure 7 The surface shows the approximate solution $u(x, t)$ of problem (23)-(24) for $\alpha=1, \beta=2$. 


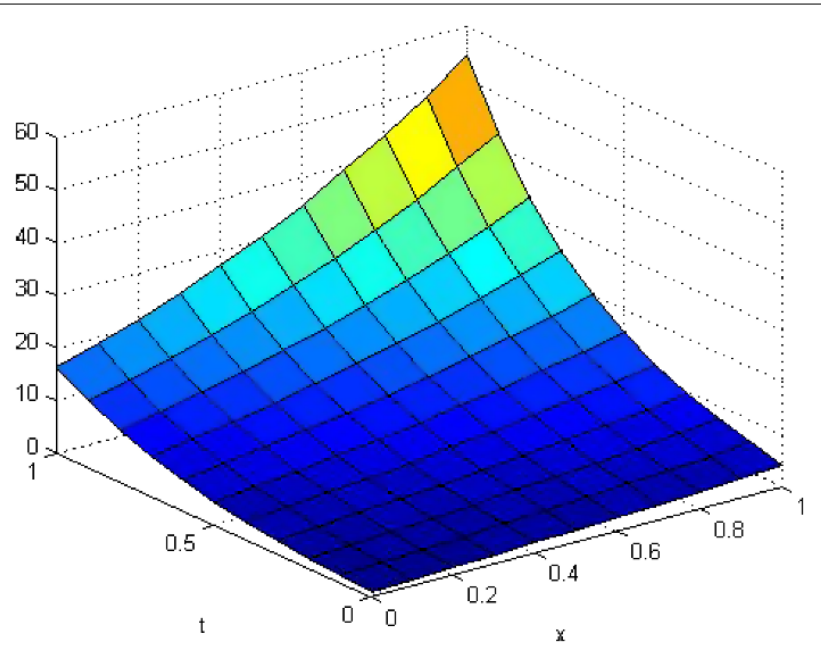

Figure 8 The surface shows the approximate solution $u(x, t)$ of problem (23)-(24) for $\alpha=0.75, \beta=1.5$.

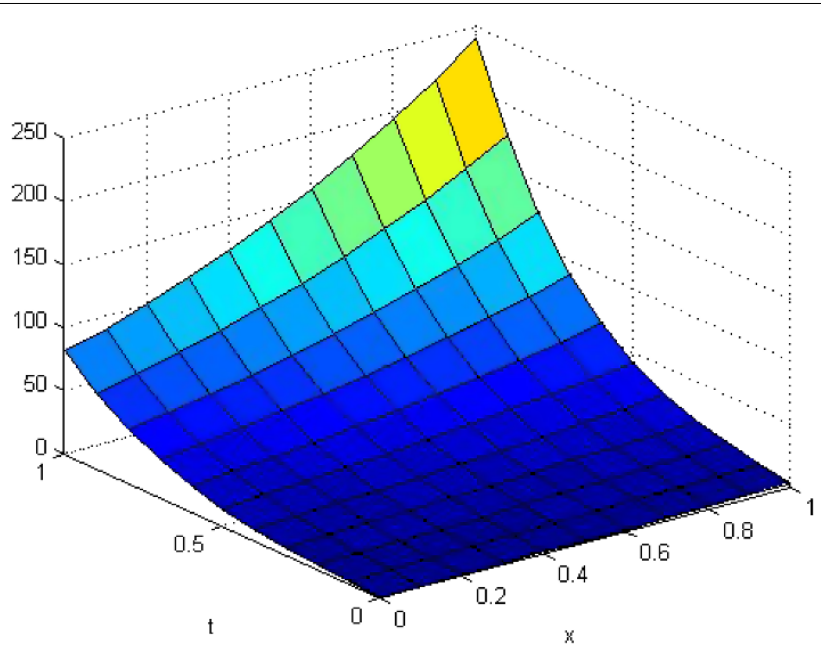

Figure 9 The surface shows the approximate solution $u(x, t)$ of problem (23)-(24) for $\alpha=0.5, \beta=1.75$.

\section{Competing interests}

The authors declare that they have no competing interests.

\section{Authors' contributions}

The authors declare that the study was realized in collaboration with the same responsibility. All authors read, checked and approved the final manuscript.

\section{Author details}

'Department of Mathematics, Kocaeli University, Umuttepe, Izmit, Kocaeli 41380, Turkey. ${ }^{2}$ Ardahan University, Ardahan, 75000 , Turkey.

\section{Acknowledgements}

Dedicated to Professor Hari M Srivastava.

The research was supported by parts by the Scientific and Technical Research Council of Turkey (TUBITAK).

Received: 19 December 2012 Accepted: 11 March 2013 Published: 30 March 2013

\section{References}

1. Oldham, KB, Spanier, J: The Fractional Calculus. Academic Press, New York (1974)

2. Podlubny, I: Fractional Differential Equations. Academic Press, San Diego (1999) 
3. Kilbas, AA, Srivastava, HM, Trujillo, JJ: Theory and Applications of Fractional Differential Equations. Elsevier, Amsterdam (2006)

4. He, JH: Nonlinear oscillation with fractional derivative and its applications. In: International Conference on Vibrating Engineering'98, Dalian, China, pp. 288-291 (1998)

5. $\mathrm{He}, \mathrm{JH}$ : Some applications of nonlinear fractional differential equations and their approximations. Bull Sci Technol 15, 86-90 (1999)

6. He, JH: Approximate analytical solution for seepage flow with fractional derivatives in porous media. Comput Methods Appl Mech Eng 167, 57-68 (1998)

7. Metzler, R, Klafter, J: The random walk's guide to anomalous diffusion: a fractional dynamics approach. Phys Rep 339, 1-77 (2000)

8. Gazizov, RK, Kasatkin, AA, Lukashchuk, SY: Group-invariant solutions of fractional differential equations. In: Tenreiro Machado, JA, Luo, ACJ, Barbosa, RS, Silva, MF, Figueiredo, LB (eds.) Nonlinear Science and Complexity, 1st edn., vol. 1, pp. 51-61. Springer, Dordrecht (2011)

9. Huang, F, Liu, F: The time-fractional diffusion equation and fractional advection-dispersion equation. ANZIAM J. 46, $1-14(2005)$

10. Huang, F, Liu, F: The fundamental solution of the space-time fractional advection-dispersion equation. J. Appl. Math Comput. 18(2), 339-350 (2005)

11. Momani, S: Non-perturbative analytical solutions of the space- and time-fractional Burgers equations. Chaos Solitons Fractals 28(4), 930-937 (2006)

12. Odibat, Z, Momani, S: Application of variational iteration method to nonlinear differential equations of fractional order. Int. J. Nonlinear Sci. Numer. Simul. 1(7), 15-27 (2006)

13. Das, S: Solution of fractional vibration equation by the variational iteration method and modified decomposition method. Int. J. Nonlinear Sci. Numer. Simul. 9, 361 (2008)

14. Momani, S, Odibat, Z: Numerical comparison of methods for solving linear differential equations of fractional order. Chaos Solitons Fractals 31(5), 1248-1255 (2007)

15. Odibat, Z, Momani, S: Approximate solutions for boundary value problems of time-fractional wave equation. Appl. Math. Comput. 181(1), 767-774 (2006)

16. Yildirim, A: An algorithm for solving the fractional nonlinear Schrödinger equation by means of the homotopy perturbation method. Int. J. Nonlinear Sci. Numer. Simul. 10,445-451 (2009)

17. Ganji, ZZ, Ganji, DD, Jafari, H, Rostamian, M: Application of the homotopy perturbation method to coupled system of partial differential equations with time fractional derivatives. Topol. Methods Nonlinear Anal. 31, 341 (2008)

18. Hang, X, Shi-Yun, L, Xiang-Cheng, Y: Analysis of nonlinear fractional partial differential equations with the homotopy analysis method. Commun. Nonlinear Sci. Numer. Simul. 14, 1152-1156 (2009)

19. Saha, RS, Bera, RK: The random walk's guide to anomalous diffusion: a fractional dynamics approach. Phys. Rep. 339 1-77 (2000)

20. Odibat, Z, Momani, S: Numerical methods for nonlinear partial differential equations of fractional order. Appl. Math. Model. 32, 28-39 (2008)

21. El-Sayed, AMA, Gaber, M: The Adomian decomposition method for solving partial differential equations of fractal order in finite domains. Phys. Lett. A 359, 175-182 (2006)

22. Sheu, LJ, Tam, LM, Lao, SK: Parametric analysis and impulsive synchronization of fractional-order Newton-Leipnik systems. Int. J. Nonlinear Sci. Numer. Simul. 10, 33-44 (2009)

23. Xu, C, Wu, G, Feng, JW, Zhang, WQ: Synchronization between two different fractional-order chaotic systems. Int. J. Nonlinear Sci. Numer. Simul. 9, 89-95 (2008)

24. Yildirim, A: Homotopy perturbation method for solving the space-time fractional advection-dispersion equation. Adv. Water Resour. 32, 1711-1716 (2009)

\section{Submit your manuscript to a SpringerOpen ${ }^{0}$ journal and benefit from:}

- Convenient online submission

- Rigorous peer review

- Immediate publication on acceptance

- Open access: articles freely available online

- High visibility within the field

- Retaining the copyright to your article

Submit your next manuscript at $>$ springeropen.com 